\title{
Calidad de vida relacionada con salud bucal en niños y ansiedad dental de sus cuidadores. Revisión de la literatura
}

\section{Oral health-related quality of life in children and dental anxiety of their caregivers Literature review}

\author{
Jorge García-Cárdenas (c) ${ }^{1 a}$, Gabriela Silva-Oyarzún (b) ${ }^{1 a}$, Ricardo Cartes-Velásquez (b)
}

\section{RESUMEN}

Se ha logrado determinar que la calidad de vida, así como también los hábitos que nosotros podamos ir adquiriendo durante la vida, pueden afectar de alguna u otra forma nuestra calidad de vida, la salud oral, como por ejemplo los hábitos alimenticios, las diferencias socioeconómicas y raciales/étnicas, educación de los padres y la estructura familiar, así como también el miedo que proyectan los padres o tutores por experiencias previas a sus hijos que recién están incursionando en la atención dental ya en un nivel más consciente. El objetivo de este artículo es revisar conceptos elementales de la calidad de vida relacionada con salud bucal en niños y su relación con la ansiedad dental de sus cuidadores.

Palabras Clave: Calidad de vida; Salud bucal; Ansiedad dental; Niños. (Fuente: DeCS BIREME)

\section{ABSTRACT}

It has been determined that the quality of life, as well as the habits that we may acquire during life, can affect in one way or another our quality of life, oral health, such as eating habits, socioeconomic differences and racial / ethnic, parental education and family structure, as well as the fear that parents or guardians project from previous experiences to their children who are just entering dental care on a more conscious level. The objective of this article is to review elementary concepts of oral health- related quality of life in children and its relationship with dental anxiety of their caregivers.

Key words: Quality of life; Oral health; Dental anxiety; Children. (Source: MeSH NLM)

\footnotetext{
${ }^{1}$.Facultad de Odontología, Universidad Andrés Bello, Concepción. Chile.

${ }^{2}$. Fundación Kimntrum, Concepción. Chile.

a Interno de Odontología

. Doctor en Ciencias Médicas

Correspondencia:

Este es un artículo de acceso abierto distribuido bajo la licencia Creative Commons Atribución 4.0 Internacional (CC BY 4.0)

https://creativecommons.org/licenses/by/4.0/deed.es

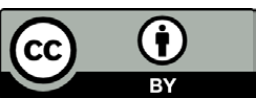

Citar como: García Cárdenas J; Silva Oyarzún G, Cartes Velásquez R. Calidad de vida relacionada con salud bucal en niños y ansiedad dental de sus cuidadores. Revisión de la literatura. KIRU. 2021; 18(2):103-109. https://doi.org/10.24265/kiru.2021.v18n2.05 


\section{INTRODUCCIÓN}

El concepto de calidad de vida (CV) ha comenzado a utilizarse cada vez más en el campo de las evaluaciones en salud o como medida de bienestar. Pese a esto, no existe una definición única del concepto ni una completa diferenciación con otros conceptos similares, siendo frecuentemente mal utilizado ${ }^{(1)}$. Su uso extendido es a partir de los sesentas, cuando los científicos sociales inician investigaciones en CV recolectando información y datos objetivos como el estado socioeconómico, nivel educacional o tipo de vivienda, siendo muchas veces estos indicadores económicos insuficientes, dado que sólo eran capaces de explicar un $15 \%$ de la varianza en la CV individual ${ }^{(1)}$.

En la década de los setentas el termino CV se usó indistintamente para nombrar numerosos aspectos diferentes de la vida, como estado de salud, función física, bienestar físico (síntomas), adaptación psicosocial, bienestar general, satisfacción con la vida y felicidad. El sentido del término CV es indeterminado, y aunque tenga un ajuste adecuado en determinadas circunstancias no deja de tener un riesgo ideológico. En su esencia parece un asunto lingüístico, cultural y fenomenológico acaso perteneciente al mundo de la filosofía y que como constructo hipotético desafía su manejo científico ${ }^{(2)}$.Para algunos autores, el concepto de CV se origina a fin de distinguir resultados relevantes para la investigación de salud, derivado de las investigaciones tempranas en bienestar subjetivo y satisfacción con la vida ${ }^{(1)}$.

Sin embrago la OMS define calidad de vida como la percepción del individuo de su lugar de vida, el contexto de su cultura y sistema de valores en los que vive en relación con sus objetivos, sus expectativas, sus normas, sus inquietudes. Se trata de un concepto que está influenciado por la salud física del sujeto, su estado psicológico, su nivel de independencia, sus relaciones sociales, así como su relación con el entorno ${ }^{(3)}$.

Para la medición de la calidad de vida se propone el conjunto de los indicadores físicos más representativos de las diferentes actividades o aspectos que la comprenden y que deben sustentarse a partir de tres condiciones: equidad, seguridad y sostenibilidad ${ }^{(4)}$.

Calidad de vida relacionada con la salud (CVRS) es, pues, el aspecto de la calidad de vida que se refiere específicamente a la salud de la persona y se usa para designar los resultados concretos de la evaluación clínica y la toma de decisiones terapéuticas. Esta utilización básica del concepto se inició en EE. UU. aproximadamente en los 80's, con la confluencia de dos líneas de investigación: una de la investigación clínica de la medición del "estado funcional" y otra la de la investigación psicológica del bienestar y la salud mental ${ }^{(2)}$. Los avances metodológicos de la psicometría y a la combinación de medidas de función física y del bienestar psíquico permitieron el desarrollo de la moderna investigación clínica de la calidad de vida relacionada con salud ${ }^{(5)}$.

Para algunos autores, la CVRS es frecuentemente identificada como una parte de la calidad de vida general, mientras que otros sugieren que su uso es a menudo intercambiable con el de CV, asumiendo así que ambos constructos estarían evaluando similares dimensiones ${ }^{(1)}$. Pese a esta discrepancia, la mayoría de los autores siguiere que la CV debe ser diferenciada de la CVRS, debido a que este último es utilizado en el campo de la medicina en su interés por evaluar la calidad de los cambios como resultado de intervenciones médicas, porque debe limitarse a la experiencia que el paciente tiene de su enfermedad, porque caracterizan la experiencia del paciente en los resultados de los cuidados médicos o para establecer el impacto de la enfermedad en la vida diaria, es decir, es una medición de la salud desde el punto de vista de los pacientes (apreciación subjetiva). Otros investigadores han sugerido que la CVRS debería enfocarse en características tales como ingreso, libertad y calidad del medioambiente ya que estos indicadores están fuera de la esfera de lo que se entiende por resultados médicos, y podrían ser vinculados con mayor facilidad a estos que otros indicadores propuestos por las ciencias sociales tales como la felicidad, la satisfacción con la vida o el bienestar subjetivo ${ }^{(1)}$.

Se puede definir salud oral como un estándar de los tejidos orales que contribuye a la salud física, psicológica y social de bienestar al permitir a las personas a comer, comunicarse y sociabilizar sin molestias, vergüenza o angustia y lo que les permite participar plenamente en su desarrollo social ${ }^{(6)}$. La mayoría de las enfermedades y afecciones orales comparten factores de riesgo modificables con las principales ENT (enfermedades no transmisibles) como las enfermedades cardiovasculares, cáncer, enfermedades respiratorias crónicas y diabetes. Estos factores de riesgo incluyen el consumo de 
tabaco, alcohol y las dietas poco saludables con alto contenido de azucares libres que estan aumentando a nivel mundial. Existe una relación comprobada entre la salud oral y la salud general $^{(7,8)}$.

El objetivo de este artículo es revisar conceptos elementales de la calidad de vida relacionada con salud bucal en niños y su relación con la ansiedad dental de sus cuidadores. Para esto se consultaron las bases de datos PubMed, SciELO y Google Scholar, se utilizaron los términos de búsqueda "calidad de vida relacionada con salud bucal", "ansiedad dental", "miedo dental" y "fobia dental", seleccionando preferentemente artículos publicados en el periodo 2010-2020 con población infantil que incluyera datos clínicos y psicológicos de niños y sus padres.

\section{Calidad de vida relacionada con salud oral en niños}

A pesar de su aparición relativamente reciente en las últimas décadas, la calidad de vida relacionada con la salud oral (OHRQoL, por sus siglas en inglés) tiene implicaciones importantes para la práctica clínica de la odontología y la investigación dental. La OHRQoL es una parte integral de la salud en general y el bienestar y es reconocido por la OMS como un segmento importante del Programa Mundial de Salud Oral.

Las campañas internacionales de salud utilizan estrategias de publicidad y marketing para mejorar el bienestar al retratar imágenes positivas de salud oral que representan valores de salud globales. Los esfuerzos van desde la eliminación del dolor hasta la iluminación de imágenes estéticas utilizando sonrisas "atractivas" con dientes "blancos". La OHRQoL se ha aplicado ampliamente como una medida de impacto de las enfermedades y trastornos orales en individuos y la sociedad. A diferencia de los indicadores clínicos normativos, las medidas de calidad de vida apuntan a identificar amplias consecuencias del mal manejo en cuanto a salud oral desde la perspectiva de adultos, niños y familias que se ven afectadas. Además de considerar la autopercepción del individuo acerca de su salud también incluye aspecto sociales, emocionales y funcionales, abarcando al sujeto de una forma íntegra y no sólo tomando en cuenta aspectos como el dolor o la alteración funciona ${ }^{(9)}$. Por lo que este concepto es multifactorial en donde predomina la autopercepción de las condiciones orales y su impacto en el desempeño diario, considerando al paciente como un sujeto integral, incluyendo la apreciación del mismo en cuanto a su salud ${ }^{(10)}$.

A las medidas de OHRQoL se han incorporado el seguimiento de enfermedades, facilitando así la comparación de morbilidad entre las condiciones de salud con el potencial de guiar a gran escala y el establecimiento de prioridades, $(11,12)$ permitiendo: monitorear la salud poblacional, evaluar las políticas públicas en salud, focalización de recursos según necesidades, diagnóstico, y evaluar severidad, tratamiento, impacto y pronóstico de las diferentes enfermedades, teniendo como ventaja la evaluación individual de cada paciente.

La mala salud bucal se ha reconocido cada vez más como una causa importante de impacto negativo en el rendimiento diario y calidad de vida $^{(13)}$. Los problemas causados por enfermedades orales afectan directamente en la calidad de vida de las personas. Uno de estos incluye la dificultad en la masticación, generando una menor ingesta de alimentos y en consecuencia pérdida de peso, insomnio, irritabilidad y baja autoestima, en los niños esto también influye en un menor rendimiento escolar (11). Dentro de las enfermedades más frecuentes en la cavidad oral están la caries dental y enfermedad periodontal, que no solo se desarrollan de forma física, sino que también generan consecuencias económicas, sociales y psicológicas, además de generar dolor, incomodidad y otras condiciones que afectan a la estética y por ende la autoestima del paciente (11)

El ambiente en el cual viven los niños y crecen influencian tanto su comportamiento como hábitos en salud y su autopercepción de la salud oral ${ }^{(13)}$. Existen investigaciones que demuestran que el estado de la salud bucal en niños está relacionado con el ingreso, estado socioeconómico, educación de los padres y estructura familiar, entre otros; por lo que se ha establecido que las personas con bajos niveles económicos tienen más probabilidades de verse expuestas a varios factores de riesgo los cuales afectan a la salud bucal y la salud general. En cambio, las familias con más alto nivel socioeconómico tienen mayor accesibilidad $y$ con mayor rapidez a la atención dental, debido a que pueden acudir a una consulta privada, por lo que se ven mayormente beneficiados ${ }^{(14)}$.

Por otra parte, los adultos responsables al tener menor nivel educativo tienen menos conocimientos sobre lo que significa caries y ésta la pueden ver como algo normal e inevitable 
durante la primera infancia y asi desetimar el impacto que generan las condiciones orales en relación con otros factores o prioridades que ellos consideran mas importantes. Al igual que las creencias, actitudes y percepciones del cuidador sobre su salud bucal se asocian al estado dental del menor ${ }^{(9)}$. Se ha logrado reportar que las niñas a diferencia de los niños tienen un mayor OHRQoL. La asociación entre sexo y salud oral relacionada con la calidad de vida se pueden explicar debido a que las niñas tienen mayor conciencia en cuanto a salud bucal, salud general y estética ${ }^{(13)}$. Otro factor significativo que también está asociado a menor nivel socioeconómico es el hacinamiento, ya que se observó que, en hogares de más de tres niños, estos tenían un menor valor de OHRQoL, dado que los padres aparte de preocuparse de sustentar un hogar, de los quehaceres hogareños, y priorizar sus recursos económicos en mantener dicho hogar, tiene que dividir el tiempo restante entre sus hijos, y no le dedicaban la atención necesaria a la salud oral (14).

\section{Ansiedad dental}

El miedo y la ansiedad dental (MAD) se refiere a fuertes sentimientos negativos que están asociados con el tratamiento dental. La prevalencia informada de esta situación entre niños y adolescentes de diferentes países oscila entre 5 a $33 \%{ }^{(15)}$. Actualmente no existe una explicación concreta para el desarrollo de MAD en niños y su etiología aún no se comprende por completo ya que está relacionado con múltiples factores, como la edad, antecedentes socioculturales y experiencias dentales de sus padres ${ }^{(16)}$.

Los niños con MAD intentan retrasar el tratamiento dental, también demuestran poca cooperación durante las visitas al dentista, lo que compromete los resultados del tratamiento. La continuación de esta problemática durante la infancia puede seguir hasta la vida adulta y ser un predictor significativo para evitar la atención dental en edad adulta ${ }^{(15)}$. Las consecuencias de esta problemática van desde pobre salud oral hasta problemas psicosociales que conllevan a una baja autoestima en el individuo, ${ }^{(17)}$. por lo que prevenir e interceptar este miedo y ansiedad se considera un enfoque crítico para mejorar la salud bucal y a la vez su calidad de vida en el presente y para el futuro ${ }^{(15)}$.

Se ha sugerido que los padres juegan un papel importante en la enseñanza de sus hijos para lidiar con estas situaciones adversas. En general, el entorno familiar, factores como la crianza parental y el estilo de apego contribuyen a la gravedad de los síntomas de ansiedad en niños ${ }^{(18)}$. Diversos estudios han observado que el MAD de los padres/tutores podría ejercer una influencia sobre el MAD de los niños, ya que muchos adultos con MAD pueden verbalizar sus sentimientos de miedo frente a sus hijos, creando una impresión negativa frente al tratamiento dental. Además, existen diversos factores que influencian el desarrollo emocional y cognitivo del niño y en consecuencia de esto su comportamiento. El apego es uno de los vínculos iniciales entre los progenitores/tutor y el niño, por lo que mientras más fuerte sea este, mayor será la influencia de los padres o tutores sobre el menor ${ }^{(19)}$. Por lo que el estilo de apego contribuye a la severidad de los síntomas de MAD en los niños ${ }^{(20)}$.

También existe evidencia preliminar de que el comportamiento del preescolar en la consulta dental está relacionado con la inteligencia emocional de la madre, ya que esto tiene relación con la habilidad que va a tener el niño para manejar condiciones estresantes. A su vez se ha encontrado relación entre el desarrollo cognitivo y las reacciones del niño frente a procedimientos restauradores dentales, los cuales están influenciados por el tipo de crianza que recibe por parte de sus padres/tutores ${ }^{(19)}$. Este último factor influye en el tipo de estrategias de afrontamiento que tiene el preescolar, especialmente estrategias de evitación y estrategias agresivas durante situaciones estresantes cotidianas. El estilo de crianza autoritario se relaciona con niños más ansiosos (20).

La relación madre-hijo es uno de los parentescos más importantes que el individuo experimenta en toda su vida. Por lo que es importante mencionar el rol fundamental de la madre durante el tratamiento odontológico. Muchos estudios han evaluado el MAD de las madres $y$ estos señalan que las madres ansiosas pueden transmitir emociones poco constructivas para sus hijos, interfiriendo negativamente en la experiencia propia del niño frente a la situación odontológica ${ }^{(21)}$.

También se ha relacionado el nivel de ansiedad de los padres/tutores con el nivel socioeconómico, ya que se ha demostrado que, en familias con ingresos superiores a dos sueldos mínimos, las madres presentaban un 
nivel leve de ansiedad, Esto se puede explicarpor la mayor facilidad de acceso a la información de prevención, procedimientos dentales y visitas al odontopediatra en comparación a familias de menor ingresos económicos; ${ }^{(22)}$ en cambio, las familias con bajos ingresos económicos tienden a tener menor alfabetización en salud en general, lo que conlleva a tener una menor capacidad de obtener, procesar y comprender información básica de salud y servicios, necesarios para tomar decisiones de salud apropiada lo que se traduce a una mayor ansiedad dental. En consecuencia, padres/tutores con mayor nivel de MAD y menor ingreso económico, son menos propensos a llevar a sus hijos a la consulta dental, postergando así tratamientos dentales de prevención, teniendo como resultado, niños con más problemas bucales y que deben ser sometidos a tratamientos muchos más invasivos (23)

Otro factor que puede estar involucrado en el MAD es el orden de nacimiento, este podría determinar parcialmente la capacidad que van a tener los niños para afrontar el estrés en situaciones médicas. Se ha descubierto que los primogénitos tienen un MAD más alto $\mathrm{y}$ son menos cooperativos durante la atención dental (25). Existen otras investigaciones, en las cuales se observa que los niños en edad preescolar con tres o más hermanos tienen niveles más altos de MAD, la explicación para esto es que los niños de familias más grandes pueden estar expuestos de manera directa o indirectamente a información sobre los tratamientos dentales $u$ observar comportamientos ansiosos de sus hermanos durante la atención dental ${ }^{(19)}$.

La edad del menor igual se puede ver relacionada, ya que hay investigaciones que informan que el MAD podría disminuir a medida que aumenta la edad, debido a que el menor va adquiriendo una mayor capacidad cognitiva, una mejor comprensión de su entorno y una mejor percepción de situaciones estresantes. Además, los niños en edad preescolar asimilan de mejor manera las explicaciones dadas por sus padres/tutores y/o dentista lo que generaría una disminución de MAD ${ }^{(16)}$. Los niños de menor edad pueden presentar mayor MAD debido a que tienden a tener más miedo a lo desconocido y a la separación de sus padres durante la atención dental ${ }^{(22)}$. La influencia de la edad puede ser explicada por la inmadurez del desarrollo psicológico del niño, según la teoría de Piaget, los niños más pequeños en el periodo pre-operacional (desde los 2 hasta los
6 años) no tienen la capacidad de hacer frente a los procedimientos dentales, por lo que pueden estar más propensos a la adquisición de miedos y problemas en el manejo de la conducta durante la visita al dentista ${ }^{(21)}$.

En la literatura se pueden encontrar algunos estudios que evidencian que el nivel de ansiedad en niñas es más alto en comparación a los niños. $Y$ en relación con los padres o tutores se exhibe que las mujeres presentan mayor nivel de ansiedad que los hombres y generalmente son las madres o tutoras quienes acompañan al menor en la consulta ${ }^{(24)}$.

Además, es vital el reconocer la importancia de la etapa preescolar, en este tema, debido a que es en esta etapa en que los niños comienzan a adquirir rasgos de personalidad de sus padres o tutores, a quienes consideran como modelos por lo que es muy probable que internalicen valores, actitudes y visiones de sus padres/tutores que gradualmente se van a ir convirtiendo en su propio sistema de creencias ${ }^{(15)}$. Reconocer todos estos factores que generan, desencadenan 0 acrecientan dicho MAD, ayudaría en gran medida al profesional en cómo abordar al paciente, con el fin de obtener un grado de colaboración adecuada de parte de este $^{(25)}$.

\section{MAD y OHRQOL}

A pesar del evidente impacto de factores psicosociales de la familia, especialmente de los cuidadores, sobre la salud bucal y OHRQoL de los niños, la literatura sobre esta materia es escasa. Con todo, el MAD aparece como un predictor fuerte y significativo en OHRQoL, ya que influyen negativamente en la $\mathrm{CV}$ de los individuos. Se debe tener en cuenta que esta relación probablemente sea bidireccional, ya que un OHRQoL pobre también puede causar o exacerbar el MAD, generando como consecuencia visitas irregulares al dentista, un alto número de caries y un mayor patrón de evitación $^{(26)}$. Las personas con una baja OHRQoL y que además sufren de MAD, presentan una menor satisfacción con su apariencia facial y dental, vergüenza relacionada con el estado bucal, relacionado a la estética, dolor y algún tipo de disfunción ${ }^{(27)}$.

Existe un círculo vicioso entre MAD y OHRQoL, dado que comienza con la evitación del cuidado dental, seguido de un posterior deterioro de la salud oral, lo que genera múltiples consecuencias. 
Las personas que ingresan a este círculo vicioso buscan ayuda cuando el problema dental ya existe y/o causa dolor, o algún tipo de discapacidad funcional ${ }^{(27)}$. Dejar de ir al odontólogo, puede provocar la evolución a un estado de gravedad mayor, de un problema bucal que era en su inicio simple, y por ende con el pasar del tiempo exige un tratamiento más especializado, generalmente procedimientos invasivos y traumáticos en las que muchas veces provoca la pérdida de piezas dentarias ${ }^{(21)}$. En consecuencia, el MAD se ve aumentado, debido a que los individuos se deben enfrentar a situaciones de mayor estrés, y a su vez disminuyendo la OHRQoL (27). EI MAD además de causar un deterioro de la salud dental también a su vez está asociado a sentimientos de menor bienestar en general, vitalidad, culpa, vergüenza, depresión, aislamiento social, menor satisfacción y calidad de vida ${ }^{(28)}$.

EI MAD parental tiene una correlación importante con OHRQoL en los niños, ya que este puede llegar a generar una barrera importante para acceder al cuidado dental. Esta se puede generar, por ejemplo, por la evitación que tienen los padres/tutores en asistir a la consulta dental por el MAD, lo que va retrasando las visitas de sus hijos; o debido al comportamiento de los mismos, que se puede ver influenciado por los comentarios negativos de sus padres frente a la atención odontológica.

El retraso a tratamientos odontológicos generará consecuencias en la salud de los preescolares, y como se ha estudiado, los menores son propensos a una variedad de trastornos orales que pueden comprometer la funcionalidad, bienestar y calidad de vida. ${ }^{29} \mathrm{Se}$ ha informado que niños con un nivel alto de MAD, presentan una disminución en su bienestar emocional, debido a su estado de salud bucal; de modo que el MAD de los padres/tutores, actitudes y las percepciones, pueden afectar no solo su propia salud dental, sino que también la de sus hijos ${ }^{(29)}$.

Por lo tanto, el reconocimiento temprano y el manejo del MAD durante las primeras etapas de la infancia y niñez, es esencial para brindar un tratamiento eficaz, reducir la evitación dental, reducir problemas de manejo del comportamiento, mejorando la salud bucal y la OHRQoL hacia la vida adulta ${ }^{(26)}$.

\section{Conclusiones}

EI MAD juega un rol relevante, no solo en la evitación de los cuidados de la salud bucal a través de la visita regular al dentista, sino en el estado de salud bucal misma y consecuentemente en la OHRQoL; esto se manifiesta en un círculo vicioso entre MAD, mala salud bucal y bajos de niveles de OHRQoL. Si bien existe evidencia sobre esta relación, aún faltan estudios con mejor nivel de evidencia que caractericen esta relación más acabadamente y ofrezcan alternativas de tratamiento para la población atrapada en este círculo vicioso.

\section{REFERENCIAS BIBLIOGRÁFICAS}

1. Urzúa A, Caqueo-Urízar A. Calidad de vida: una revisión del concepto. Ter Psi. 2012; 30(1): 61- 71

2. Fernández-López $J$, Fernández-Fidalgo $M$, Cieza A. Quality of life, health and wellbeing conceptualizations from the perspective of the International Classification of Functioning, disability and health (ICF). Rev Esp Salud Pública 2010; 84(2): 169-84.

3. Tipán L, López R, Borges S, Cabrera M. Adaptación transcultural y validación del Early childhood oral health impact scale en preescolares ecuatorianos. Rev Odontol. 2017; 19(1): 75-88.

4. Robles-Espinoza Al, Rubio-Jurado B, De la Rosa-Galván EV, Nava-Zavala $\mathrm{AH}$. Generalidades y conceptos de calidad de vida en relación con los cuidados de salud. El Residente 2016; 11(3): 120-125.

5. Najman JM, Levine S. Evaluating the impact of medical care and technologies on the quality of life: A review and critique. Soc Sci Med. 1981; 15:107-115.

6. Thomson $\mathrm{W}$, Broder $\mathrm{H}$. Oral health related quality of life in children and adolescents. Pediatric Clin. 2018; 65(1):1073-1084.

7. Cartes-Velásquez R, Araya E, Valdés C. Maloclusiones y su impacto psicosocial en estudiantes de un liceo intercultural. Int $\mathrm{J}$ Odontostomatol. 2010;4(1):65-70.

8. Ruiz XM, Abara CV, Cartes-Velásquez R. Impacto de la salud bucal en la calidad de vida de escolares de 11 a 14 años, Licantén, 2013. Revista Clin Period Implantol Rehab Oral. 2014;7(3):142-148.

9. Chaffee $\mathrm{C}$, Rodriguez $\mathrm{P}$, Kramer $\mathrm{P}$, Vítolo $M$, Feldens $C$. Oral health-related qualityof-life scores differ by socioeconomic status and caries esperience. Community Dent Oral Epidemiol. 2017; 45(3):216-224.

10. Vintimilla N, Del castillo C. Calidad de vida relacionada a la salud bucal en escolares 
de Sayausí, Cuenca Ecuador. Rev Estom. 2017; 27(4):227-34.

11. Spanemberg J, Cardoso J, Slob E, LópezLópez J. Quality of life related to oral health and its impact in adults. J Stomatol Oral Maxillofac Surg. 2019;120(3):234-239

12. Vintimilla N, Del castillo C. Calidad de vida relacionada a la salud bucal en escolares de Sayausí, Cuenca Ecuador. Rev Estom. 2017; 27(4):227-34.

13. Piovesan C, Ferreira J, Saraiva R, Machado T. Impact of socioeconomic and clinical factors on child oral health-related quality of life (COHRQoL). Qual Life Res. 2010; 19:1359-1366

14. Kumar S, Kroon J, Lalloo R. A systematic review of the impact of parental socioeconomic status and home environment characteristics on children's oral health related quality of life. Health Qual Life Outcomes. 2014; 12-41

15. Wu L, Gao X. Children's dental fear and anxiety: exploring family related factors. BMC Oral Health. 2018; 18:100

16. Abanto J, Vidigal E, Carvalho T, SÁ S, Bönecker M. Factors for determining dental anxiety in preschool children with severe dental caries. Braz Oral Res. 2017; 31

17. Shahnavaz S, Rutley S, Larsson K, Dahllöf G. Children and parent's experiences of cognitive behavioral therapy for dental anxiety - a qualitative study. Int J Clin Pediatr Dent. 2015; 25: 317-326

18. Krikken J, Veerkamp J. Child rearing styles, dental anxiety and disruptive behavior; an exploratory study. Eur Arch Paediatr Dent. 2008; 9:23-28.

19. Díaz D, Gaete P. Ansiedad del niño, de su tutor, del operador y el comportamiento que presenta el paciente en la clínica odontopediátrica. [tesis] Viña del mar: Universidad Andrés Bello; 2016

20. Krikken J, van Wijk A, ten Cate J, Veerkamp J. Child dental anxiety, parental rearing style and referral status of children. Community Dent Health. 2012; 29(4): 28992
21. Munayco E, Mattos-Vela M, Torres G, Blanco D. Relación entre ansiedad, miedo dental de los padres y colaboración de niños al tratamiento odontológico. ODOVTOS-Int. J. Dental Sc. 2018; 20(3): 81-91

22. Busato P, Garbin R, Santos C, Paranhos L, Rigo L. Influence of maternal anxiety on child anxiety during dental care: crosssectional study. Sao Paulo Med J. 2017;135(2):116-22

23. Shin $W$, Braun $T$, Inglehart $M$, Habil $P$. Parent's dental anxiety and oral health literacy: effects on parent's and children's oral health-related experiences. J. Public Health Dent. 2013;74(2014): 195-201

24. Assunção $C$, Losso $E$, Andreatini $R$, de Menezes J. The relationship between dental anxiety in children, adolescents and their parents at dental environment. J Indian Soc Pedod Prev Dent. 2013; 31(3): 175-179

25. Bartolomé $B$, Torres $L$, Méndez $M$, Almenara M. Factores que influyen en la ansiedad dental del paciente infantil. Cient. Dent. 2019; 16(1): 59-66

26. Merdad L, El-Housseiny A. Do children`s previous dental experience and fear affect their perceived oral health-related quality of life (OHRQoL)?. BMC Oral Health. 2017; $17: 47$

27. Carlsson V, Hakeber M, Boman U. Associations between dental anxiety, sense of coherence, oral health-related quality of life and health behavior- a national Swedish cross-sectional survey. BMC Oral Health. 2015; 15:100

28. Levin L, Zini A, Levine J, et al. Dental anxiety and oral health-related quality of life in aggressive periodontitis patients. Clin Oral Invest. 2018; 22:1411-1422

29. Buldur B, Guvendi O. Conceptual modelling of the factors affecting oral health-related quality of life in children: A path analysis. Int J Paediatr Dent. 2019; 30: 181-192.
Jorge García-Cárdenas

ORCID iD: (1) http://orcid.org/0000-0001-7986-1599

coke.garcia@live.cl

Gabriela Silva--Oyarzún

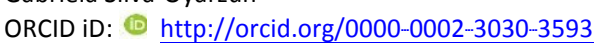

gaby.silvaoyarzun@gmail.com
Ricardo Cartes--Velásquez

ORCID iD: (1) http://orcid.org/0000--0001--5831--7224

cartesvelasquez@gmail.com

Copyright $($ La revista. La revista Kiru es publicada por la Facultad de Odontología de la Universidad de San Martin de Porres, en Lima, Perú. 